\title{
EFFECT OF RF PHASE NOISE ON THE SSC BEAM
}

\author{
J.A. Ellison \\ Department of Mathematics and Statistics \\ The University of New Mexico \\ Albuquerque, New Mexico 87131 \\ B.S. Newberger \\ Institute for Fusion Studies \\ The University of Texas at Austin \\ Austin, Texas 78712 \\ H.-J. Shih \\ Superconducting Super Collider Laboratory* \\ Dallas, Texas 75237
}

\begin{abstract}
In this paper we investigate the effect of three types of $\mathrm{rf}$ phase noise on the SSC beam: white, low pass and notched noise. We present and compare results from Monte-Carlo tracking simulations using a linear transfer map and from numerical solutions of an associated diffusion equation.
\end{abstract}

\section{Introduction}

Particle diffusion in longitudinal phase space due to if noise, which leads to emittance growth and particle lose, has been observed in the $\operatorname{SppS}[1]$. It is therefore important to understand the effect of if noise on the SSC beam so that the noise tolerance level of the collider if system can be specified and a feedback system to minimize noise can be designed. Dôme[2] has provided a good theoretical basis in which the effect of noise is described by a Markov diffusion process. In our work, the associated Fokker-Planck equation is numerically integrated by the method of lines[3]. For comparison and additional information on the diffusion process, computer simulations in which the proton bunch circulates in a linear lattice with noise injected into the rf cavity are also carried out.

This work originated in studying a very promising application of bent crystal channeling to extract the SSC beam. This is attractive because a bent crystal of small length can channel particles through a relatively large angle without incurring excessive particle loss. To exploit this phenomenon, a small part of the circulating beam must be gradually brought onto the crystal septum. It is in this respect that injection of white phase noise into the if system is considered and, when combined with the introduction of high dispersion at the location of the bent crystal, has been found feasible[4]. However, white noise appears to perturb the beam core in a way that might affect collider operations. Thus two other types of $\mathrm{rf}$ phase noise, low pass and notched, which are expected to diffuse particles primarily from the beam tail, are explored. A detailed account of computer simulations using low pass noise in connection with bent crystal extraction is given in [5].

\section{Review of Theory}

The underlying theory is reviewed in this section and readers are refered to [2] for more details. For a sinusoidal rf voltage

* Operated by the Univervities Research Association, for the U.S. Department of Energy under Contract No. DE-AC02-89ER40486.

U.S. Government work not protected by U.S. Copyright. with phase noise, the turn-to-turn energy and phase variations in a stationary bucket are given by

$$
\begin{gathered}
P_{n+1}-P_{n}=-K_{0} \sin \phi_{n}, \\
\phi_{n+1}-\phi_{n}=P_{n+1}+\varphi_{n+1}-\varphi_{n}
\end{gathered}
$$

with $P_{n}=2 \pi h \eta\left(\Delta p_{n} / p_{1}\right), K_{0}=2 \pi h \eta\left(e V / p_{1} v_{1}\right)$ and $\eta=\alpha_{c}-$ $1 / \gamma^{2}$. Here $n$ is the turn number, $\phi$ the actual phase with noise relative to the synchronous phase, $\varphi$ the phase noise, $h$ the harmonic number, $\alpha_{c}$ the momentum compaction factor, $\gamma$ the ratio of the particle's total energy to its rest energy, $p$. (v.) the momentum (velocity) of the synchronous particle, $e$ the electron charge, $V$ the amplitude of the if voltage and $\Delta$ refers to a difference taken with respect to the synchronous particle. It can be shown that $K_{0}=\Omega^{2} T_{0}^{2}$ where $\Omega$ is the angular frequency of small synchrotron oscillations and $T_{0}$ the revolution period. In the continuous time approximation, the finite difference Eqs. (1) and (2) are replaced by the differential equations

$$
\begin{gathered}
\dot{P}=-\Pi^{2} \sin \phi, \\
\dot{\phi}=P+\dot{\varphi}(t)
\end{gathered}
$$

where $P \equiv P_{n} / T_{0}$. These two equations can be derived from the time-dependent Hamiltonian

$$
H=\frac{1}{2} P^{2}+\Omega^{2} \cdot 2 \sin ^{2} \frac{\phi^{2}}{2}+P \dot{\varphi}(t) .
$$

In the unperturbed case $(\varphi=0)$, the action $J=$ $(1 / 2 \pi) \oint P d \phi$ is conserved. For $\varphi \neq 0$, Dôme assumes conditions are such that the action evolves by a Markov diffusion process and then derives the one-dimensional diffusion equation

$$
\frac{\partial \rho}{\partial t}=\frac{\partial}{\partial J}\left(D(J) \frac{\partial \rho}{\partial J}\right)
$$

with diffusion coefficient

$$
D(J)=4 \sum_{m=1,3, \ldots}^{\infty} \frac{\left(m \omega_{\bullet}\right)^{4}}{\cosh ^{2}(m v)} S_{\varphi}\left(m \omega_{\nu}\right),
$$

for the evolution of the action density $\rho=\rho(J, t)$. Here $\omega_{\text {. }}$ is the angular frequency of the unperturbed synchrotron oscillation. The spectral density $S_{\varphi}(\omega)$ is the Fourier transform of the autocovariance $R(\tau)=\langle\varphi(t) \varphi(t+\tau)\rangle$ of the stationary process $\varphi(t)$ and the noise variance is simply $\sigma_{\varphi}^{2}=R(0)$. The quantities $J, \omega_{\lrcorner}(J)$ and $v(J)$ are easily defined through the 
intermediate variable $k, 0 \leq k<1$, by $J=\left(8 \cap k^{2} / \pi\right) B(k)$, $\omega_{s}=\Omega(\pi / 2 K(k))$ and $v=(\pi / 2) K\left(\sqrt{1-k^{2}}\right) / K(k)$ where $K$ is the complete elliptic integral of the first kind and $B=$ $\int_{0}^{\pi / 2} \cos ^{2} \psi d \psi / \sqrt{1-k^{2} \sin ^{2} \psi}$. The variable $k$ is related to the unperturbed energy $H_{0}$ by $H_{0}=2 k^{2} \Omega^{2}$.

\section{Monte-Carlo Tracking Simulations}

One thousand protons are followed in a linear lattice for the SSC. To each proton six independent variables are assigned: horizontal coordinate $(x)$ and angle $\left(x^{\prime}\right)$, vertical coordinate $(y)$ and angle $\left(y^{\prime}\right)$, path length deviation from the reference trajectory $(l)$ and fractional momentum deviation from the synchronous momentum $\left(\delta=\Delta p / p_{1}\right)$. The initial values of each are randomly generated according to Gaussian distributions with the rms values of $x, x^{\prime}, y$ and $y^{\prime}$ determined by the lattice functions and the rms values of $l$ and $\delta$ determined by assuming an energy spread of $1 \mathrm{GeV}$. To carry the protons around, a one-turn map constructed by the lattice functions is applied to the vector $\left(x, x^{\prime}, y, y^{\prime}, l, \delta\right)$. When the protons traverse the rf cavity, the relative momentum difference is changed according to

$$
\delta_{n+1}=\delta_{n}-\frac{e V}{p_{0} v_{1}} \sin \left(2 \pi h \frac{l_{n}}{C_{0}}+\varphi_{n}\right)
$$

where $C_{0}$ is the SSC circumference. Equation (8) is derived from (1) by observing that $\phi_{n}=(2 \pi h) l_{n} / C_{0}+\varphi_{n}$.

In computer simulations white noise refers to the case where the $\varphi_{x}$ are drawn randomly from a Gaussian distribution each time the bunch passes through the rf cavity. To obtain low pass and notched noise, the following is done beforehand: generate a time series of white noise for $N$ turns, $\left\{\varphi_{1}, \varphi_{2}, \cdots, \varphi_{N}\right\}$, compute the discrete Fourier series $\left\{\Phi_{1}, \Phi_{2}, \cdots, \Phi_{N}\right\}$ and construct

$$
\hat{\varphi}_{n}=F_{1} \frac{2}{N} \sum_{m=2}^{m_{0}} \operatorname{Re}\left(\Phi_{m} e^{2 \pi i(m-1)(n-1) / N}\right)
$$

in the case of low pass noise or

$$
\hat{\varphi}_{n}=F_{2}\left\{\varphi_{n}-\frac{2}{N} \sum_{m=m_{0}+1}^{m_{1}} \operatorname{Re}\left(\Phi_{m} e^{2 \pi i(m-1)(n-1) / N}\right)\right\}
$$

in the case of notched noise. Here $m_{0} \geq 2$ is the largest integer such that $m_{0} \leq Q_{\text {.o }} N \alpha, m_{1}$ the smallest integer such that $m_{1} \geq Q_{\text {.o }} N, Q_{\text {.a }}$ the small-oscillation synchrotron tune, $\alpha$ the filtering fraction and $F_{1}$ and $F_{2}$ are normalization factors such that $\varphi_{n}$ and $\hat{\varphi}_{n}$ have the same $\sigma_{\varphi}$ (For the SSC, $\Omega=26.6$ $\mathrm{rad} / \mathrm{sec}$ and $\left.Q_{\circ o}=0.00123\right)$. Since $n$ is the turn number and the synchrotion tune for $\Phi_{m} \exp (2 \pi i(m-1)(n-1) / N)$ is $Q_{m}=$ $(m-1) / N$, Eqs. (9) and (10) say that low pass noise contains only the terms whose synchrotron tunes are less than $\alpha Q_{\text {.o and }}$ notched noise contains all the terms except those whose tunes

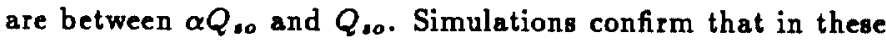
two cases the particles in the core, with tunes greater than $\alpha Q_{. \circ}$, are much less perturbed than in the case of white noise. This is demonstrated in Figs. 1 and 2 which show the initial distributions in longitudinal phase space of the lost particles in the presence of white noise with $\sigma_{\varphi}=0.06$ for $5.3 \times 10^{\circ}$ turns and in the presence of notched noise with $\sigma_{\phi}=0.155$ for $10^{\dagger}$ turns respectively. The same plot for a low pass case can be found in [5]. In the simulations, $\alpha=0.85$ and a particle is defined to be lost when it crosses the orbit whose $\delta_{\max }$ is $96 \%$ of the separatrix's. The total number of lost particles is 160 in Fig. 1 and 134 in Fig. 2.

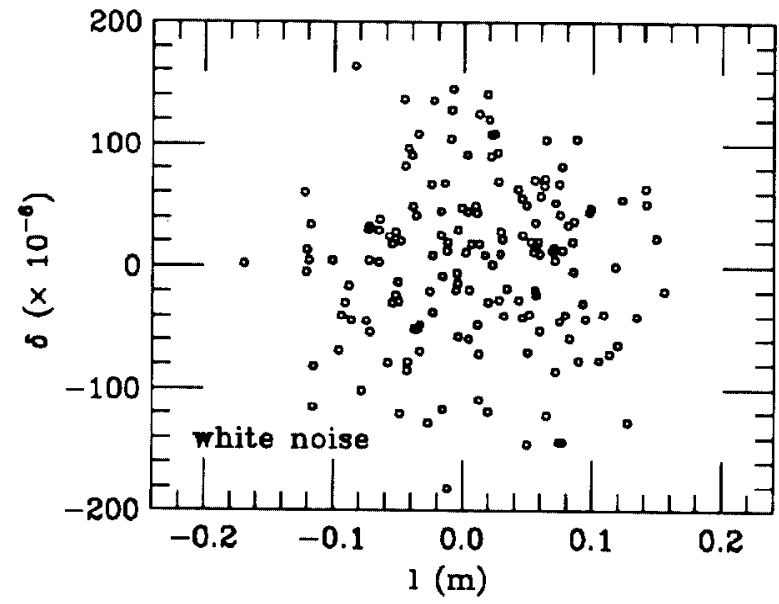

Figure 1: Initial distribution in longitudinal phase space of the lost particles in the presence of white noise.

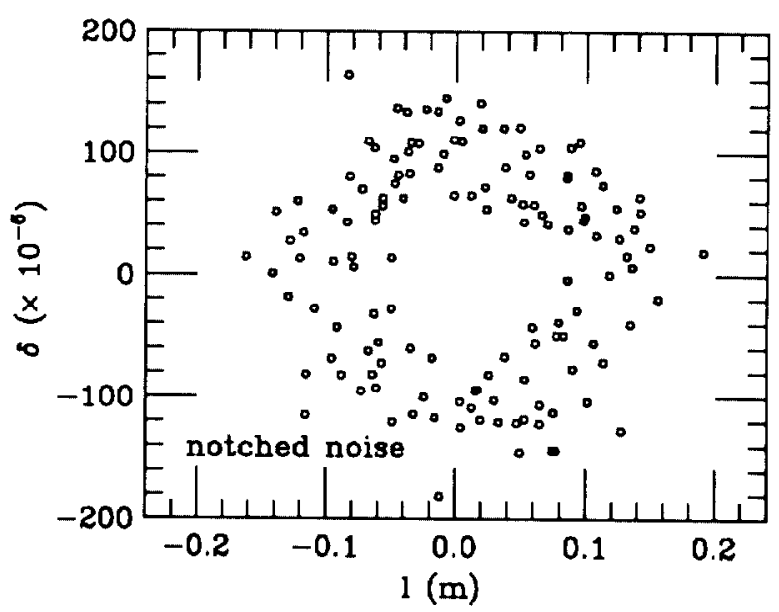

Figure 2: Initial distribution in longitudinal phase space of the lost particles in the presence of notched noise.

\section{Numerical Solutions of Diffusion Equation}

The method of lines[3] is used to integrate the diffusion equation (6). That is, the action variable $J$ is discretized, the partial derivatives with respect to $J$ are calculated by a finite difference approximation and an ODE integrator with automatic error control is employed to obtain the evolution in time of the action density at each grid point of $J$. Before integrating (6) one must first calculate the diffusion coefficient $D$ using Eq. (7) for a particular spectral density of phase noise and define proper boundary conditions. The following spectral densities are assumed for the three types of phase noise we are considering: (1) $S_{\varphi}^{\text {white }}=$ constant for $0<\omega<\infty$, (2) $S_{\varphi}^{\text {low pase }=}$ constant for $0<\omega<\alpha \Omega$ and 0 elsewhere, (3) $S_{\varphi}^{\text {notehed }}=0$ for $\alpha \Omega<\omega<\Omega$ and constant elsewhere. For white noise, an analytical expression for $D$ can be derived from Eq. (7):

$$
D_{w h i t e}=\frac{8}{\pi^{2}} \Omega^{4} S_{\varphi} k^{2} K(k)\left(B(k)-4 k^{2} B_{1}(k)\right)
$$

where $B_{1}=\int_{0}^{\pi / 2} \sin ^{2} \psi \cos ^{2} \psi \sqrt{1-k^{2} \sin ^{2} \psi} d \psi$. For low pass 
noise, $D$ is computed according to

$$
D_{\text {low mat. }}=4 \omega_{*}^{4} S_{\varphi} \sum_{m=1}^{n_{1}} \frac{(2 m+1)^{4}}{\cosh ^{2}((2 m+1) v)}
$$

where $n_{1}$ is an integer such that $\left(2 n_{1}-1\right) \omega_{\text {, }} \leq \alpha \Omega<\left(2 n_{1}+1\right) \omega_{\text {a }}$. For notched noise, $D$ is computed according to

$$
D_{\text {notched }}=D_{w k i t e}-4 \omega, S_{\bullet} \sum_{m=n_{1}}^{n_{2}} \frac{(2 m+1)^{4}}{\cosh ^{2}((2 m+1) v)}
$$

where $n_{2}$ is an integer such that $\left(2 n_{2}-1\right) \omega_{1} \leq n<\left(2 n_{2}+\right.$

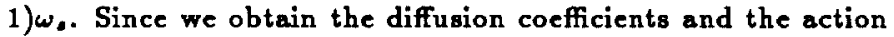
as functions of $k$, we spline fit $D(k)$ versus $J(k)$ so as to obtain $D$ as a function of $J$. With $x=J / J_{b}$ where $J_{b}=J(k=$ $0.96)$, Fig. 3 shows $D(x)$ for white, low pass and notched noise. The initial condition for Eq. (6), i.e., the initial distribution in action, is determined from the initial values of $l$ and $\delta$ that have been randomly generated in the tracking simulation and the boundary condition is taken to be absorbing at $J=J_{b}$, i.e., $\rho\left(J_{b}, t\right)=0$. The final distributions in action from Dòme's diffusion theory and the tracking simulation are compared in Fig. 4 for white noise with $\sigma_{\downarrow}=0.2$ and in Fig. 5 for notched noise with $\sigma_{\varphi}=0.155$. The final time corresponds to $10^{\circ}$ turns in the case of white noise and $3 \times 10^{6}$ turns in the case of notched noise. The agreement between simulation and theory is quite good in both cases. Since the diffusion coefficient for low pass noise is zero for $x<x_{0}$ where $x_{0}=x(\omega=\alpha \Omega)$, the beam core should not be affected. Our simulation also confirms this.

\section{Conclusions}

We found good agreement between simulation and Dome's theory on the diffusion process of the SSC beam due to if phase noise. We showed that the beam core is much less perturbed by low pass and notched noise than by white noise. Future work will include a study of amplitude noise and the effect of a feedback loop on if noise.

\section{Acknowledgements}

The authors thank A. Chao, T. Murphy and S. Peggs for many fruitful discussions and W.E. Schiesser for kindly providing us with his method of lines code DSS/2. J.A.E. gratefully acknowledges conversations with $R$. Cogburn.

\section{References}

[1] D. Boussard, "RF Techniques for $p \bar{p}^{n}$, in CERN Report No. 84-15 Accelerator School on Antiprotons for Colliding Beam Facilities, 1984, pp. 261-290.

[2] G. Dome, "Theory of RF Acceleration and RF Noise", in CERN Report No. 84-15 Accelerator School on Antiprotons for Colliding Beam Facilities, 1984, pp. 215-260.

[3] W.E. Schiesser, The Numerical Method of Lines Integration of Partial Differential Equations, Academic Press, San Diego, 1991.

[4] B. Newberger and H.-J. Shih, "Low Intensity Beam Extraction at the SSC", SSCL Report No. SSCL-344, January 1991.

[5] H.-J. Shih and A.M. Taratin, "Bent Crystal Extraction of the SSC Beam with RF Noise Induced Diffusion", SSCL Report No. SSCL-389, March 1991.

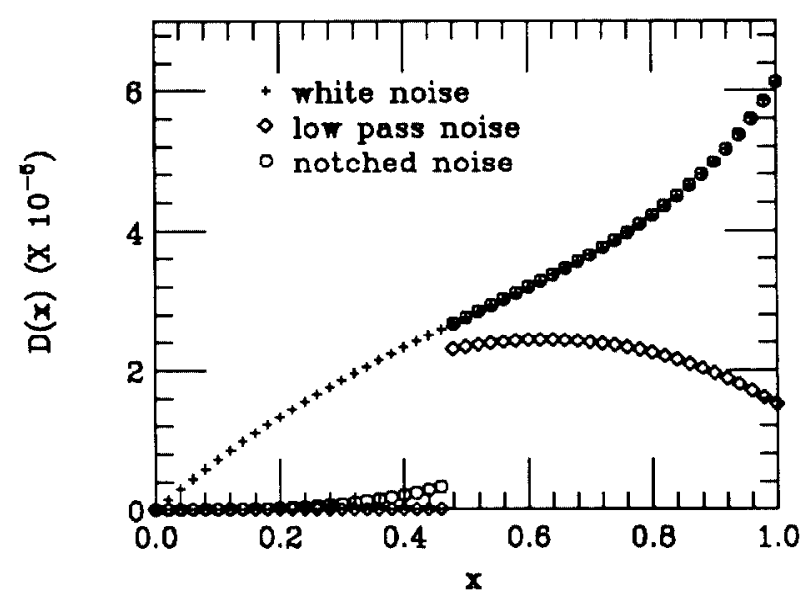

Figure 3: Diffusion coefficients for white, low pass and notched noise.

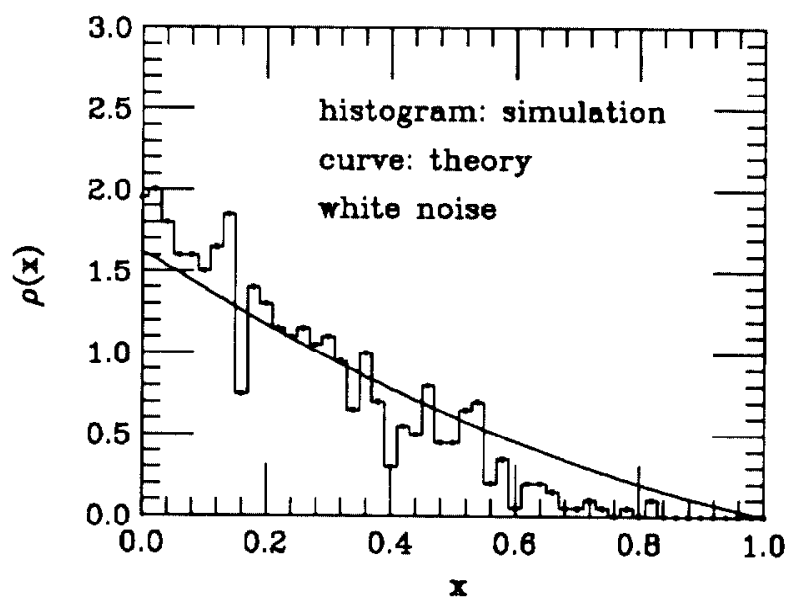

Figure 4: Final distribution in action in the presence of white noise with $\sigma_{\varphi}=0.2$ for $10^{6}$ turns.

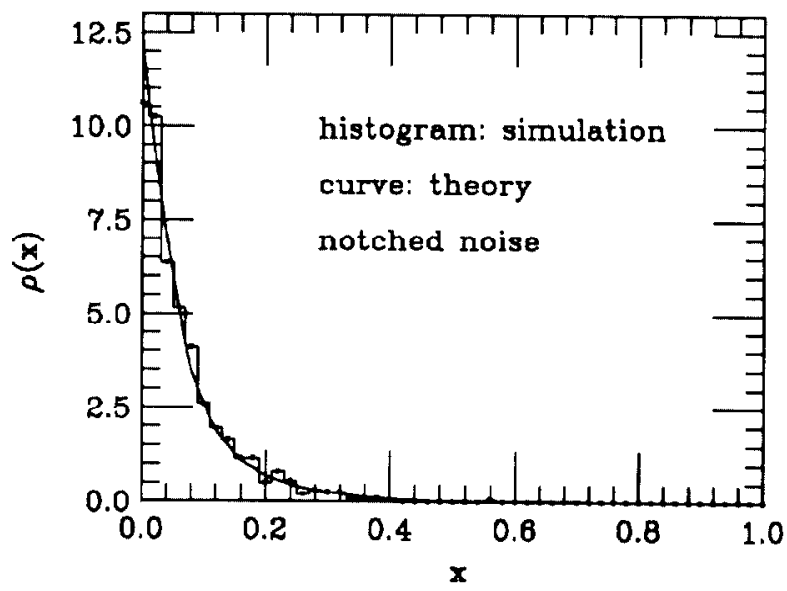

Figure 5: Final distribution in action in the presence of notched noise with $\sigma_{\varphi}=0.155$ for $3 \times 10^{6}$ turns. 